\section{Colour in the Dogfish, Scyllium canicula}

Since the completion of a paper on colour change in the dogfish ${ }^{1}$, two points of interest have arisen. The first concerns the colour cells and their natural responses in the young fish, and the second one concerns melanophore control in the various members of the group Elasmobranchii.

The colour cells of a young dogfish (S. canicula) hatched in the Zoology Department at Liverpool were investigated, and observations made on the natural responses of the melanophores. There proved to be four types of colour cells, only three of which have been described from the adult ${ }^{1}$. The natural melanophore responses to illuminated dark and light backgrounds were the same as in the adult fish ${ }^{1}$.

In the newly hatched fish, focusing from the lower skin levels to the surface on the pectoral fin, there appear in order very conspicuous deep yellow cells capable of completely rounding off and of expansion to a stellate condition, greyish brown cells never seen in the contracted state but always with blunt irregular branches, light brown melanophores and finally, dark superficial melanophores. The yellow cells are numerous and very conspicuous, whereas in the adult they are very few and frequently cannot be found at all. The greyish brown type of cell has not previously been described from either the young ${ }^{2}$ or adult ${ }^{1}$ fish. The pigment from both these latter cells was dissolved out in alcohol. The two types of melanophore are exactly similar to those described from the adult fish ${ }^{1}$.

Although on some occasions no melanophore response could be obtained, as a rule on an illuminated white background and in complete darkness the melanophores contracted, while on an illuminated black background they expanded. The melanophore response to illuminated dark and light backgrounds is, therefore, the same as in the adult ${ }^{1}$, and the same as in the young ${ }^{3}$ and adult ${ }^{4}$ of Mustelus. The response to complete darkness is not known for the adult Scyllium or for the young or adult of Mustelus. The changes of the yellow cells and of the greyish brown cells have not been studied and the ultimate fate of the latter is not known. Unfortunately, the fish died.

The observations recently made by Parker on the colour change mechanism of Raja erinacea ${ }^{5}$ and Squalus acanthias ${ }^{6}$ direct attention afresh to the possibility of a lack of uniformity in the mechanism of colour control among the Elasmobranchii.

In all Elasmobranchs so far investigated ${ }^{1,7,8}$ it is established that the normal dark background reaction (dark skin, expanded melanophores) is due to the activity of neuro-intermediate lobe of the pituitary. Where more detailed information is available, it is clear that the neuro-intermediate lobe exerts its influence by means of a blood-circulated hormone ${ }^{\mathbf{1}, \boldsymbol{0}}$.

Regarding the mechanism of the natural light background reaction (contracted melanophores), however, there is no uniformity of opinion. In Mustelus canis, Parker and Porter ${ }^{10}$ and Parker ${ }^{11}$ have described and figured paling effects in dark fins as a result of faradic stimulus and of cuts made transverse to the fin rays. These effects have been interpreted by them as showing that the natural paling response in this fish is due to direct nervous action on the melanophores. On the other hand, cuts made by $\mathrm{me}^{12}$ in the fin of a dark Scyllium canicula failed to give any paling effects, and severance of selected nerves by Young ${ }^{13}$ in Scyllium sp. and by Wykes ${ }^{14}$ in Scyllium canicula, Raia brachyrura and Raia maculata did not influence the melanophore condition or, in the latter cases ${ }^{14}$, the melanophore response in the area supplied by these nerves. Further, electrical stimulation of nerves ${ }^{14}$ in Raia brachyura, Rhina squatina and Scyllium catulus did not result in any melanophore changes. From these negative results it has been concluded that there is no direct nervous control of melanophores in these fish.

In Scyllium canicula ${ }^{1}$, Raia maculata and Raia brachyura? removal of the anterior lobe of the pituitary alone destroys the natural light background reaction, and from this it has been concluded that the natural paling response of these fish is in some way connected with the activity of the anterior lobe. The effect of removal of the anterior lobe alone on the light background response of Mustelus canis unfortu. nately has not been described.

The above facts have led me to suggest ${ }^{15}$ that in the various members of Elasmobranchii there may be found different methods of melanophore control. This suggestion has now received considerable support from the observations of Parker himself that cuts made in the fins elicit no paling response whatsoever in Raja erinacea ${ }^{5}$ and only a very uncertain response in Squalus acanthias.

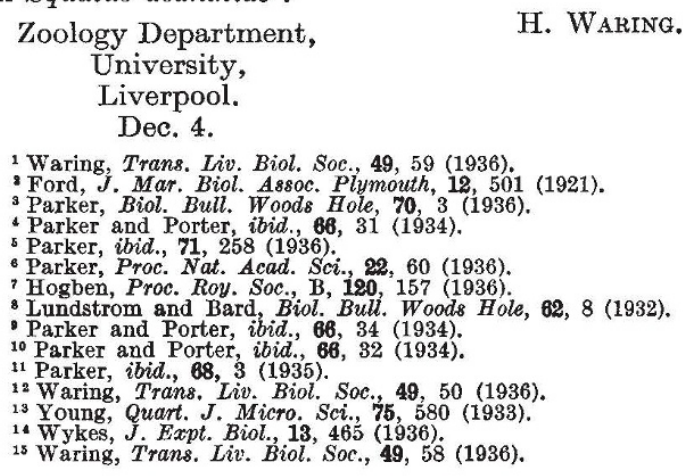

The 'Specific Action' of Ultra-Short Wireless Waves

WE have received a letter from Prof. W. Szy. manowski, of the Institute for Physiological Research at Moscow, in which he makes certain comments on our article on the above subject which recently appeared in NATURE ${ }^{1}$. We should like to be allowed to summarize these and to reply to them as briefly as possible.

(1) Prof. Szymanowski points out that his work with Dr. Hicks on the action of short waves on toxins and bacteria was begun, and a first note published $^{2}$ in 1930 , before the corresponding work by Haase and Schliephake ${ }^{3}$ (published 1931), so that our reference to the former work as confirmatory of the latter was likely to create a false impression in regard to priority. We admit the justice of this criticism, and tender our apologies to Prof. Szymanowski.

(2) In referring to two papers by Szymanowski and Hicks ${ }^{4,5}$, dealing with the action of ultra-short waves on various bacteria, toxins, etc., we stated that in the second paper they withdrew their earlier results on toxin. Prof. Szymanowski takes exception to this statement, and makes it clear that no such withdrawal was intended. We accept his correction, and would merely remark that our impression was perhaps excusable in view of the general tone of the second paper, which, in contrast to the first, was mainly devoted to the presentation of negative evidence relating to the "specific effect", and included the sentence (p. 471) : "The meagre character, however, of the action on diphtheria toxin and the delicacy of the conditions under which it may be 